\title{
AutoDispNet: Improving Disparity Estimation With AutoML
}

\author{
Tonmoy Saikia \\ Yassine Marrakchi \\ Arber Zela \\ Frank Hutter \\ University of Freiburg \\ Germany \\ \{saikiat, marrakch, zelaa, fh, brox\}@cs.uni-freiburg.de
}

Thomas Brox

\begin{abstract}
Much research work in computer vision is being spent on optimizing existing network architectures to obtain a few more percentage points on benchmarks. Recent AutoML approaches promise to relieve us from this effort. However, they are mainly designed for comparatively smallscale classification tasks. In this work, we show how to use and extend existing AutoML techniques to efficiently optimize large-scale U-Net-like encoder-decoder architectures. In particular, we leverage gradient-based neural architecture search and Bayesian optimization for hyperparameter search. The resulting optimization does not require a largescale compute cluster. We show results on disparity estimation that clearly outperform the manually optimized baseline and reach state-of-the-art performance.
\end{abstract}

\section{Introduction}

Compared to the state of computer vision 20 years ago, machine learning has enabled more generic methodologies that can be applied to various tasks rather than a single toy problem. A convolutional neural network can be trained on all sorts of classification problems, and a convolutional encoder-decoder network with skip connections can be set up for a large selection of high-resolution computer vision tasks, such as semantic segmentation, optical flow, superresolution, and depth estimation, to name just a few. With this generic methodology in place, why are there more than 5000 submissions to each computer vision conference? What do they contribute?

In practice, the methodology is not as generic as it looks at first glance. While a standard encoder-decoder network may give a reasonable solution for all these problems, results can be improved significantly by tweaking the details of this design: both the detailed architecture and several training hyperparameters, such as the learning rate and weight decay. For example, in the context of disparity estimation, manually optimizing the architecture of the original

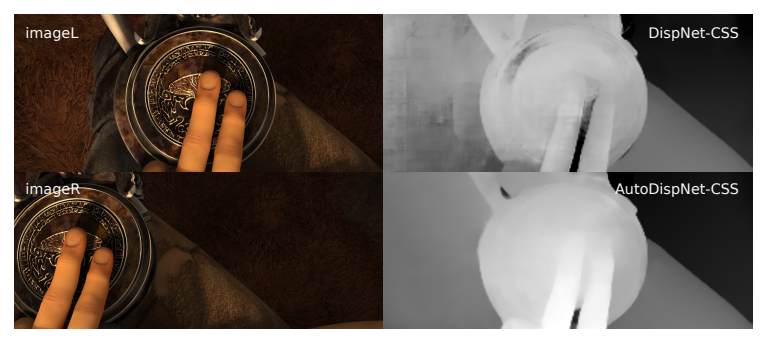

Figure 1: We present AutoDispNet-CSS, a disparity estimation network built using state-of-the-art AutoML techniques which significantly improves over the manually tuned DispNet-CSS architecture.

DispNet [52] halved the error [37]. Other works on disparity estimation found other tweaks that also improved the accuracy [44, 16, 66]. While effective, this common practice of manual architecture and parameter search contradicts the paradigm of machine learning, namely to replace manual optimization by numeric optimization.

AutoML [35] in general and automated neural architecture search (NAS [24]) in particular promise to relieve us from the manual tweaking effort. In principle, an independent validation set is enough to optimize the architecture and the hyperparameters of the learning method. Unfortunately, many of these AutoML methods have extreme computational demands. For this reason, they have been mostly applied to rather small-scale classification tasks, preferably on CIFAR, where a single network can be fully trained within a few hours. Even on such small tasks, some approaches report hundreds of GPU days to finish optimization. For large-scale encoder-decoder networks, such as DispNet, this is prohibitive.

However, there are also more efficient AutoML approaches. Although they have not yet been applied to encoder-decoder architectures, they have the potential to do so. One of them, on which we will build in this paper, is DARTS [47]. Its main idea is to have a large network that 
includes all architectural choices and to select the best parts of this network by optimization. This can be relaxed to a continuous optimization problem, which, together with the regular network training, leads to a bilevel optimization problem. Thanks to its gradient based optimization, DARTS is very efficient. However, DARTS only allows the optimization of the architecture but not the training hyperparameters.

For the latter, we propose to use an efficient black-box optimization method called BOHB [26], which builds on an efficient variant of Bayesian optimization for informed sampling of the search space. While it is somewhat more costly than DARTS, it is also entirely flexible with regard to the hyperparameter search space. We suggest to run BOHB on the architecture optimized by DARTS to train it with optimal hyperparameters.

We compare the optimized network to the already welltweaked version of DispNet [37] to investigate who is more successful in tweaking: the student or the numerical optimization procedure.

\section{Related work}

Encoder-decoder architectures have led to substantial improvements in several computer vision tasks like semantic segmentation [48, 59, 3, 29, 18, 19] and flow estimation [21, 36, 37, 56, 68]. Pioneering works which apply learning to disparity estimation consist of extending classical methods like SGM [30] with metrics learned by CNNs [73, 50, 62]. The first end-to-end network for disparity estimation is DispNet [52], which builds on FlowNetC [21]. Based on rectified stereo images, a correlation layer computes a cost volume which is further processed by the network. [37] and [54] expand DispNetC for much better performance. The extensions consist mainly of stacking multiple networks and connecting them in a residual fashion. These networks share the encoder-decoder architecture. The first module, the encoder, extracts highlevel information by gradually downsampling the feature maps while the decoder progressively produces outputs at increasing resolutions.

To reduce the effort dedicated to designing neural networks, neural architecture search (NAS) has been an active area of research in the last few years [24]. Early attempts train a recurrent neural network that acts as a metacontroller using reinforcement learning techniques [4, 81]. It learns to generate sequences encoding potential architectures by exploring a predefined search space. The same strategy is adopted in many follow-up works [6, 13, 14, 69. 78, 79, 82. Alternatively, a set of works rely on evolutionary algorithms [67, 46, 53, 57, 58, 70]. The best architecture is extracted by iteratively mutating a population of candidate architectures. Unfortunately, both strategies require hundreds to thousands of GPU days. This restricts their use to rather small networks, and research progress is limited by availability of large compute clusters.

Speed-up techniques like hypernetworks, network morphisms and shared weights lead to substantial reduction of the search cost. Hypernetworks [10, 75] generate weights for candidate networks and evaluate them without training them until convergence. Network morphisms [12, 14, 23, 25] make use of the previous learned weights to initialize new candidate architectures, thereby speeding up the performance estimation procedure. Sharing weights [55] among potential networks decreases the search time by two orders of magnitude. Multi-fidelity optimization has also been employed in NAS [5, 26, 43, 74] by exploiting partial training of architectures at the cost of noisy evaluations. Alternatively, some works [7, 15, 47] redesign the optimization problem by training a large graph containing all candidate architectures. In [7], sub-networks are probabilistically sampled and trained for a predefined number of iterations. Orthogonally, relaxations make architectural decisions like branching patterns [1] and number of channels per layer [61] learnable via gradient descent. In case of DARTS [47], real-valued architecture parameters are jointly trained with weight parameters via standard gradient descent. Cai et al. [15] propose an memory efficient implementation similar to DARTS by adding path binarization, while [71] sample from a set of one-hot random variables encoding the architecture search space and leverage the gradient information for architectural updates by relaxing the architecture distribution with a concrete distribution [51]. Despite the diversity of NAS approaches for image classification and object detection, the extension to dense prediction tasks remains restricted. To apply NAS to semantic segmentation, Chen et al. [17] restrict the search to the small pyramid pooling component of the network and occupy 370 GPUs for a whole week. In a concurrent work, Liu et al. [45] also leverage DARTS to find an optimal architecture for semantic segmentation with reduced search cost. However, their approach does not handle skip-connections for U-Net like architectures.

\section{Hyperparameter search}

Optimizing hyperparameters for dense prediction tasks with vanilla hyperparameter optimization (HPO) [8, 9, 33, 64, 65, 27] is computationally expensive. Alternatively, we use a state-of-the-art HPO method named BOHB [26] which combines the benefits of Bayesian optimization [63] and Hyperband [43], a multi-armed bandit strategy that dynamically allocates more resources to promising configurations.

BOHB uses cheap-to-evaluate approximations $\tilde{f}(\cdot, b)$ of the objective function $f(\cdot)$ (e.g. validation error), where the so-called budget $b \in\left[b_{\min }, b_{\max }\right]$ determines the strength of the approximation. For $b=b_{\max }$, we recover the true 
objective, i.e. $\tilde{f}\left(\cdot, b_{\max }\right)=f(\cdot)$. In our application, we use the number of training iterations as a budget to cut off evaluations of poorly-performing hyperparameters early, akin to approaches based on learning curve prediction [20, 5].

Hyperband repeatedly calls the Successive Halving (SH) subroutine [38] to advance promising configurations evaluated on small budgets to larger ones. SH starts by evaluating a fixed number of configurations on the cheapest budget $b_{\min }$. After these evaluations, the best fraction of $\eta^{-1}$ of configurations (based on $\tilde{f}\left(\cdot, b_{\min }\right)$ ) advance to the next budget $\eta \cdot b_{\text {min }}$; here, $\eta$ is a parameter set to 3 by default. This procedure repeats until reaching the most expensive budget $b_{\max }$ with only a few configurations left to evaluate.

While Hyperband selects configurations to evaluate uniformly at random, BOHB replaced this choice with Bayesian optimization. Specifically, it employs a multivariate kernel density estimator (KDE) to model the densities of the best and worst performing configurations and uses these KDEs to select promising points in the hyperparameter space to evaluate next. More details about BOHB are included in the supplementary material.

\section{Differential architecture search}

While BOHB can, in principle, also be used to optimize architectural parameters [74, 60], its performance degrades compared to gradient-based approaches as the dimensionality of the search space grows. BOHB also evaluates different architectures from scratch rather than exploiting weight sharing, increasing the computational burden for neural architecture search of large-scale vision architectures to a prohibitive range.

Therefore, we tackle the neural architecture search not with BOHB, but rather use the gradient-based method DARTS [47]. It combines weight sharing and first order optimization to speed up the architecture optimization by orders of magnitude compared to brute-force blackbox optimization methods, which can require thousands of GPU days [57, 82]. We propose to leave the costly architecture search to DARTS and then optimize important other hyperparameters that cannot be integrated into DARTS by BOHB in a post-hoc step.

We review the components of the DARTS approach before we adapt DARTS to the context of full encoder-decoder architectures in Section 5 .

\subsection{Search space}

Similar to other architecture search methods [46, 57, 82], DARTS optimizes relatively small, repetitive parts of the network architecture called cells. Learned cells are stacked to generate the overall network architecture in a user defined fashion.

A cell is a directed acyclic graph (DAG) consisting of $N$ nodes. The nodes can be categorized into input, inter- mediate, and output nodes. Each node $x^{(i)}$ represents a feature map and each edge $(i, j)$ represents an operation $o^{(i, j)}$ which transforms $x^{(i)}$. DARTS assumes a cell to have two input nodes and one output node. The input nodes are the outputs of the two previous cells. The output node is obtained by concatenating the outputs of all intermediate nodes. The output of each intermediate node is computed as:

$$
x^{(j)}=\sum_{i<j} o^{(i, j)}\left(x^{(i)}\right)
$$

where $o^{(i, j)} \in \mathcal{O}$. $\mathcal{O}$ is the set of all candidate operations.

In DARTS, $\mathcal{O}$ consists of the following operations: skip connection, $3 \times 3$ average pooling, $3 \times 3$ max pooling, $3 \times 3$ and $5 \times 5$ depthwise separable convolutions, $3 \times 3$ and $5 \times 5$ dilated separable convolutions with dilation factor 2 . It also includes a special "zero" operation to indicate lack of connectivity between nodes.

For classification tasks there are two cell types: a normal cell with maintains the spatial resolution of the input and a reduction cell which reduces the spatial resolution of the input by half. The structure of standard DARTS cell is shown in Figure $2 \mathrm{a}$

\subsection{Continuous relaxation}

To make the search space continuous, DARTS uses relaxation based on the softmax function. A variable $\alpha_{o}^{(i, j)} \in$ $\mathbb{R}$ is associated with each operation $o \in \mathcal{O}$ in the edge $(i, j)$ connecting nodes $i$ and $j$. The categorical choices in each edge $(i, j)$ are then relaxed by applying the softmax nonlinearity over the $\alpha_{o}^{(i, j)}$ for all possible operations $o \in \mathcal{O}$ :

$$
S_{o}^{(i, j)}=\frac{\exp \left(\alpha_{o}^{i, j}\right)}{\sum_{o^{\prime} \in \mathcal{O}} \exp \left(\alpha_{o^{\prime}}^{i, j}\right)}
$$

We set $\bar{o}^{(i, j)}=\sum_{o \in \mathcal{O}} S_{o}^{(i, j)} o\left(x^{(i)}\right)$. This weighted average of $\left(x^{(i)}\right)$ is called "mixed operation" in the remainder of this work. Therefore, (1) becomes:

$$
x^{(j)}=\sum_{i<j} \bar{o}^{(i, j)}\left(x^{(i)}\right)
$$

With this relaxation in place, the task of architecture search is equivalent to learning the set of continuous variables $\alpha=$ $\left\{\alpha^{(i, j)}\right\}$, where $\alpha^{(i, j)}$ is a vector of dimension $|\mathcal{O}|$.

\subsection{Optimization}

Since the continuous relaxation makes the set of architecture variables $\alpha$ differentiable, we can efficiently optimize them using gradient descent. DARTS [47] proposed a first order and second order approximation. In this work, we focus on the first order approximation as the second order approximation is too costly for large architectures. 


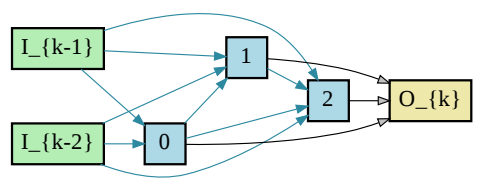

(a) Normal/Reduction cell

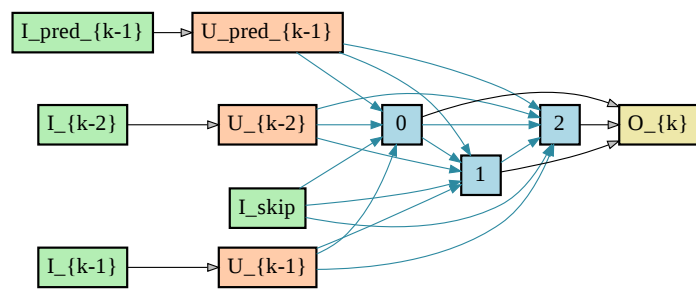

(b) Upsampling cell

Figure 2: Structure of search cells. In Figure 2a we show the structure of a normal or reduction cell. An upsampling cell is shown in Figure $2 \mathrm{~b}$. In both cases, input nodes are green, intermediate nodes are blue, output nodes are yellow. Upsampling nodes are marked as orange. A blue edge represents transformations done using mixed operations (see Section 4.2 for more details).

To solve the bilevel optimization problem, the training data is split into two disjoint subsets $\mathcal{D}_{\text {train }}$ and $\mathcal{D}_{\text {val }}$. The network parameters $w$ and architecture parameters $\alpha$ are optimized in an alternating fashion on $\mathcal{D}_{\text {train }}$ and $\mathcal{D}_{\text {val }}$, respectively, until convergence. The optimization is carried out on a search network built using stacked normal and reduction cells.

\subsection{Architecture discretization}

After training the search network to convergence, a cell structure is extracted by discretizing the continuous variables. This is achieved by retaining the top- $k$ strongest operations from all non-zero operations coming from previous nodes. The strength of an edge $(i, j)$ is set to:

$$
\max _{o \in \mathcal{O}, o \neq \text { zero }} S_{o}^{(i, j)}
$$

The extracted cells are then stacked to form a deeper network and retrained for evaluation.

\section{DARTS for dense prediction}

Dense prediction tasks involve mapping a feature representation in the encoder to predictions of larger spatial resolution using a decoder. Therefore, to apply DARTS for disparity estimation we need to extend the architecture search space such that it can support an upsampling transformation. This extension of the search space should be expressive enough to encompass common deep learning bestpractices and at the same time have enough flexibility to learn new upsampling transformations. In this section, we describe our search space and then present a search network which allows us to learn architectural cells for encoderdecoder networks.

Upsampling layers. Typically, the decoder unit of encoder-decoder networks [52, 21, 59] consists of upsampling layers which increase the spatial resolution. The most commonly used upsampling layers are transposed convolutions. Another common approach is to use billinear interpolation for upsampling followed by convolutional operations. A decoder usually has multiple decoding stages, each of which increases the spatial resolution by a factor of 2 . The number of stages depends on the downsampling factor of the encoder.

Skip connections and multi-scale refinement. Skip connections were introduced in encoder-decoder networks by [48]. They help preserve fine details in the predictions. This is achieved by concatenating the upsampled features with a feature map of the same resolution from the encoder. A coarser prediction (from the previous decoding stage), if available, is also concatenated to facilitate feature reuse. The concatenated features are then processed by convolutions to generate refined predictions. These techniques are standard for encoder-decoder networks for flow and disparity estimation [52, 21].

Upsampling cell. Several hand-designed encoderdecoder architectures have emerged [2, 28, 77, 80] which incorporate the above architecture design choices. Typically such methods propose decoding modules which apply architectural blocks (ShuffleNet [76], DenseNet [32] block, etc). However, the generic design choice of having skip connections and multi-scale refinement still remains useful in such cases. In this work, we replace such an architectural block in the decoder by a learned upsampling cell. The same DAG-based formulation for normal and reduction cells (see Section 4.1, can be used to define an upsampling cell. Our upsampling cell has four inputs : $I_{k-1}, I_{k-2}, I_{-}$pred $_{k-1}$ and $I_{\text {skip }}$. The inputs $I_{k-1}, I_{k-2}$ are the outputs of the last two preceding cells, $I_{-}$pred $_{k-1}$ represents a prediction from the previous decoding stage and $I_{\text {skip }}$ represents a feature map in the encoder obtained via skip connection. The inputs $I_{k-1}, I_{k-2}$ are upsampled by transposed convolutions whereas the input $I_{-}$pred $_{k-1}$ 


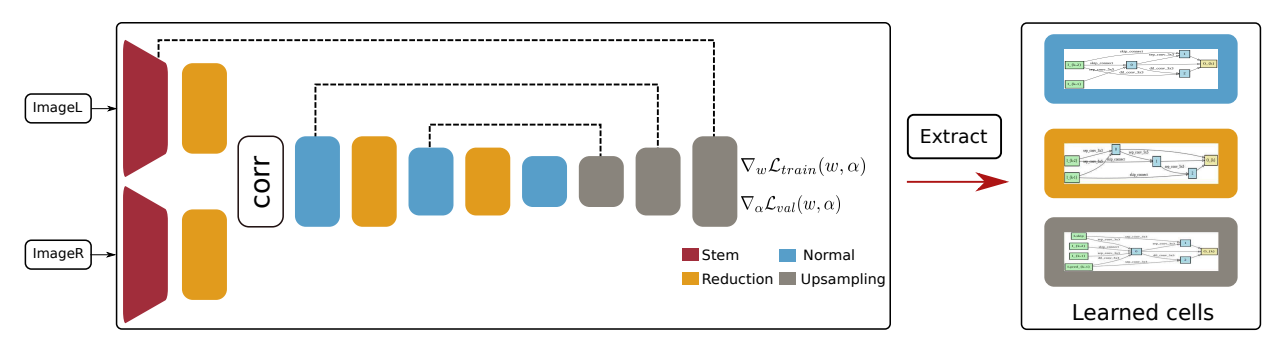

(a) Search network

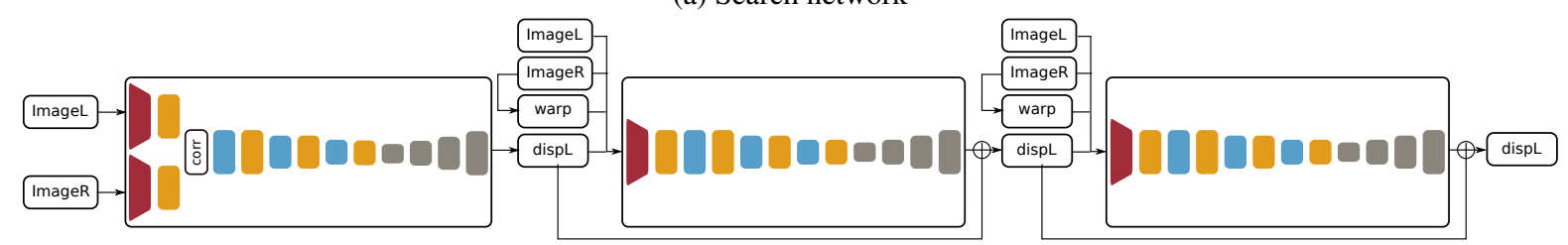

(b) AutoDispNet-CSS

Figure 3: Dense-DARTS for disparity estimation. The search network used to learn cells for disparity estimation is shown in Figure 3a Three types for cells are learned: normal, reduction and upsampling. The stem cells are simple convolutional layers with a fixed structure. It also contains a correlation layer like a standard DispNetC [52]. Skip connections from encoder to decoder are denoted by the dashed lines. After training, the three cell structures are extracted as described in Section 4.4 . Using the extracted cells, a final network (Figure 3b] is assembled using the CSS structure introduced in [37].

is upsampled by bilinear interpolation (following [37]). A schematic of the upsampling cell is shown in Figure $2 \mathrm{~b}$. The intermediate nodes in the upsampling cells process all inputs via mixed operations. To be consistent, we use the same set of operations for a mixed operation as DARTS (see Section 4.1). The outputs of all intermediate nodes are concatenated to form the output of the cell, which is then processed by a $2 \mathrm{D}$ convolution to get an upsampled disparity prediction.

Search Network. Compared to standard DARTS which is trained on CIFAR10 [41] with $32 \times 32$ images, training datasets for disparity estimation [52] consist of images which are about 500 times larger in terms of pixel amount. Therefore, to feasibly train a search network on a single GPU (Nvidia GTX1080Ti), we downsample the training images by half. Ground truth disparity values are additionally rescaled by a factor of 0.5 . The encoder part of the search network begins with a stem cell followed by stacked reduction and normal cells. The stem cell consists of two standard convolutional layers with kernel sizes $7 \times 7$ and $5 \times 5$ and stride 2 which further downsample the input. Similar to the DispNetC architecture [52, 37], the stem cell and the first reduction cell compose the Siamese part of the encoder which extract features from the left and right rectified stereo view. The extracted features are processed by a correlation layer [21]. The correlation layer performs patch comparison between the two feature maps obtained from the Siamese part of the network. Such explicit feature matching helps in significant error reduction [52]. The rest of the encoder is formed by stacking normal and reduction cells in an alternating fashion. The decoder consists of stacked upsampling cells with skip connections to the encoder. The encoder has a total of 6 cells (normal + reduction) with a final downsampling factor of 32 . The decoder consists of three upsampling cells which output predictions at different spatial resolutions. We pre-define each cell to have three intermediate nodes and initialize the first cell to have 24 channels. Each reduction cell then increases number of channels by a factor of 2. In the decoder, an upsampling cell reduces the number of channels by half with each upsampling step. An illustration of our search network is shown in Figure 3a. For training the search network, we optimize the end-pointerror (EPE) [21, 52] between the predicted and ground truth disparity maps. A loss term is added for each prediction after an upsampling step. The losses are optimized using the first-order approximation of DARTS as described in Section 4.3. We refer to our search network as Dense-DARTS.

\section{Architectures}

After training Dense-DARTS, we extract a normal, a reduction, and an upsampling cell as described in Section 4.4 . A schematic of the extraction process is shown in Figure 3a A network needs to be built using the extracted cells before it can be trained for final evaluation. In this section we introduce our baseline architecture and present network variants we consider for evaluation.

Baseline architecture. For a strong baseline we choose a recent state-of-the-art disparity estimation net- 
work, DispNet-CSS [37], which is an improved version of the original DispNet [52] manually optimized by an expert. It consists of a stack of three networks, consisting of one DispNet-C [52] and two DispNet-S [52].

Single network. To compare the performance of the extracted cells, we first build a single network for comparison with the first network in the DispNet-CSS stack. In each network the encoder downsamples the input by a factor of 64 and the output resolution of the decoder is one-fourth of the input resolution. For a fair comparison, we use seven encoder cells and four decoder cells to get the same resolutions at the bottleneck and the final layer. This network is constructed in the same fashion as the search network, as described in Section 5 , but with the extracted cells. The number of channels for the first cell $\left(C_{\text {init }}\right)$ is set to 42 , to match the number of parameters in DispNet-C. We call this network AutoDispNet-C.

Refinement with stacks. Using the same configurations as AutoDispNet-C, we construct the AutoDispNet-S architecture by replacing the Siamese part and correlation layer with a single stream of cells. In a stacked setting, the AutoDispNet-S network refines a disparity map from the previous network. Similar to [37], the input to the refinement network is a concatenation of warped image, previous disparity prediction and the rectified image pair. The first network provides an initial disparity estimate. Each following network in the stack refines the previous network's output by predicting the residual disparity. The residual disparity is added to the previous network's output to obtain a refined estimate. We denote a stacked AutoDispNet-C and two AutoDispNet-S as AutoDispNet-CSS. The full network stack is shown in Figure $3 \mathrm{~b}$

Smaller networks. We also experiment with different values of $C_{\text {init }}$ to obtain AutoDispNet architectures with different numbers of parameters. We choose a smaller variant with $C_{\text {init }}=18$ for comparison with our baseline. This configuration is denoted as AutoDispNet-css with a lowercase "c" and "s".

BOHB variants. We also use $\mathrm{BOHB}$ to tune the learning rate and weight decay of AutoDispNet architectures. We denote networks of this category by AutoDispNet-BOHB$(*)$, where $(*)$ stands for C, CS or CSS. Details about training settings are mention in Section 7.1

\section{Experiments}

\subsection{Experimental setting}

Datasets. For training our search network we use the standard FlyingThings3D [52] dataset which provides ground truth disparity maps generated using rendered scenes. The dataset consists of train and test splits with 21,818 and 4, 248 samples respectively. Each sample has a spatial resolution of $960 \times 540$.
Training Dense-DARTS. Following [47], we divide the train split of FlyingThings into two halves. The first and second halves are used to optimize the train and validation losses respectively. The test split is left untouched to evaluate the extracted architectures at a later stage. We use the same data augmentation settings as commonly used for training DispNet [52, 37]. The search network is trained by minimizing the end point error as described in Section 5 . The train loss is optimized using SGD with base learning rate of 0.025 and annealing to 0.001 using the cosine schedule [49]. To optimize the validation loss, we use the Adam optimizer [40] with a base learning rate of $1 e-4$. We add $L 2$ regularization on the weight parameters $w$ and architecture parameters $\alpha$ with factors of $3 e-4$ and $1 e-3$ respectively. Before optimizing $w$ and $\alpha$ alternatingly, we warm start the search network by optimizing only $w$ for 100k iterations. After the warm-start phase we optimize both $w$ and $\alpha$ for $200 \mathrm{k}$ iterations. We also found that annealing the softmax temperature for the mixed operation leads to slightly better results.

The extracted cells after training the search network are shown in Figure 4 Note that the search process discards all pooling operations. We also see that normal and upsampling cells (which process feature maps at the same or higher spatial resolution) include dilated convolutions, whereas the reduction cell (which downsamples feature maps) consists only of separable convolutions and some skip connections. This observation is in agreement with common usage patterns of operations for dense prediction. For instance, state-of-the art disparity estimation methods [44, 37, 68] are fully convolutional and do not contain any pooling operations. Dilated convolutions have been extensively used to obtain state-of-the art results for semantic segmentation [18, 19].

Training AutoDispNet architectures. For training the AutoDispNet-CSS stack we follow the same training procedure as our baseline architecture [37]. For training each refinement network, all previous network weights are frozen [37]. Each network is trained for $600 k$ iterations using the Adam [40] optimizer with a base learning rate of $1 e-4$. The learning rate is dropped at $300 k, 400 k, 500 k$ with a factor of 0.5 .

Hyperparameter tuning with BOHB. For AutoDispNet-C we optimize the learning rate and weight decay coefficient. Each function evaluation in BOHB involves training a network with hyperparameters sampled from a configuration space and evaluating it on a validation set. In this case, we use the test split of FlyingThings3D for validation and use Sintel as a test set. For small classification networks this usually works because training takes only a few hours. However, in our case training is expensive. Training a single network in the stack takes around 3.5 days on a single Nvidia GTX1080Ti 


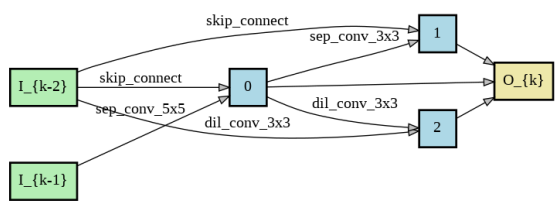

(a) Normal

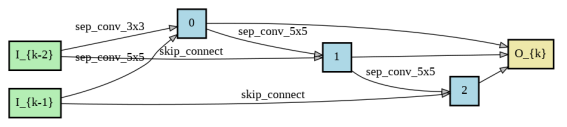

(b) Reduction

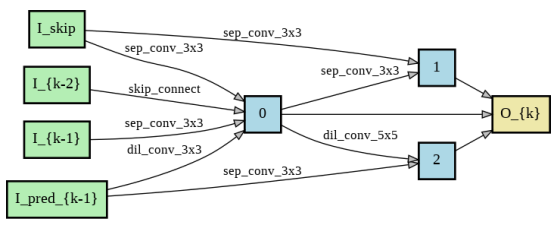

(c) Upsampling

Figure 4: Learned cells using Dense-DARTS. We visualize the extracted DAG for each cell type. $I_{k-1}$ and $I_{k-2}$ denote the two input nodes and $O_{k}$ is the output node. The numbered blue nodes depict intermediate nodes, where numbers indicate the depth at which the node was placed in the DAG before extraction.

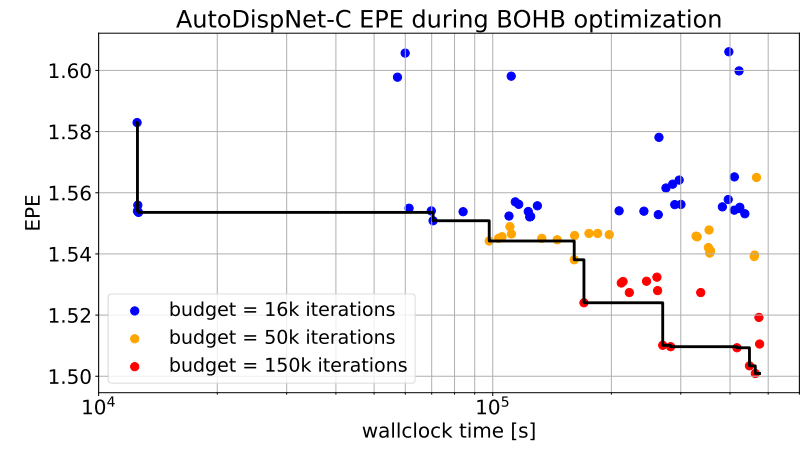

Figure 5: Hyperparameter optimization procedure. AutoDispNet-C EPE on FlyingThings3D of all sampled configurations on the different budgets throughout the BOHB optimization procedure. The black line shows the best performing configurations (incumbent) as a function of time. Note that the value on the $\mathrm{x}$ axis is the time when each evaluation finished relative to the BOHB start time, and not the training time per network.

GPU. Therefore, to make our function evaluations cheaper, we optimize the learning rate and weight decay for a restart schedule [49]. Specifically, we take a snapshot of the network after $450 k$ iterations and restart training with a learning rate sampled by BOHB. The learning rate is annealed to zero at $16.67 k, 50 k$ and $150 k$ iterations (depending on which of these budgets BOHB evaluates the sampled configurations) following a cosine function [49]. This reduces the training cost by a factor of four. The optimized hyperparameter are then used to restart training for successive networks in the stack. We found that using BOHB to tune hyperparameters for the refinement network did not boost performance (we include experimental results in the supplemental).

We ran BOHB in parallel on 5 GPU workers for a total number of 11 SuccessiveHalving iterations. We used the default BOHB settings with $\eta=3$ and budgets $16.67 \mathrm{k}, 50 \mathrm{k}$ and $150 \mathrm{k}$ mini-batch iterations. This is equivalent to 26 full function evaluations on 1 worker, i.e a total of $33.42 \mathrm{GPU}$ days. Figure 5 shows the EPE of all sampled configurations throughout the optimization procedure. As we can see, for the budgets of $16.67 \mathrm{k}$ and 50k iterations we do not notice any major improvement over time. However, for the maximum number of iterations we observe that BOHB finds a good region in the hyperparameter space and keeps sampling around that area.

\subsection{Results}

\begin{tabular}{|l|cc|cc|}
\hline Architecture & $\begin{array}{c}\text { FlyingThings3D } \\
\text { (test) }\end{array}$ & $\begin{array}{c}\text { Sintel } \\
\text { (train) }\end{array}$ & $\begin{array}{c}\text { Params } \\
\text { (M) }\end{array}$ & $\begin{array}{c}\text { FLOPs } \\
\text { (B) }\end{array}$ \\
\hline \hline DispNet-C [37 & 1.67 & 3.19 & 38 & 75 \\
\hline AutoDispNet-c & 1.98 & 3.53 & 7 & 16 \\
AutoDispNet-C & 1.53 & 2.85 & 37 & 61 \\
AutoDispNet-BOHB-C & $\mathbf{( 1 . 5 1 )}$ & $\mathbf{2 . 6 6}$ & 37 & 61 \\
\hline
\end{tabular}

Table 1: Performance of a single network. We demonstrate improved accuracy of our AutoDispNetC architecture over our baseline DispNetC. End-point errors are shown on the FlyingThings3D and Sintel datasets. The best performance is obtained by optimizing the hyperparameters with BOHB. The parentheses indicate that FlyingThings3D test split is used to optimize hyperparameters.

Single network results. Table 1 shows the result of the automatically optimized DispNet relative to the baseline. AutoDispNet yields significantly stronger numbers with about the same number of parameters. Additional hyperparameter optimization with BOHB yields another improvement on the Sintel dataset [11]. It is worth noting that the networks were only trained and optimized on the FlyingThings3D dataset, but not on any part of Sintel. This shows that the automated optimization not only overfits better to a particular dataset but improves the general capability of the network.

Stacked network results. For state-of-the-art performance on disparity estimation, it is necessary to stack multiple networks. Table 2 shows that the benefits of automated 


\begin{tabular}{|l||c|c|c|}
\hline \multicolumn{1}{|c||}{} & \multicolumn{3}{c|}{ Number of Networks } \\
\cline { 2 - 4 } & 1 & 2 & 3 \\
\hline \hline DispNet & C & CS & CSS \\
EPE & 3.19 & 2.49 & 2.36 \\
Params & 38 & 77 & 116 \\
FLOPS & 75 & 135 & 195 \\
\hline \hline AutoDispNet & c & cs & css \\
EPE & 3.53 & 2.80 & 2.54 \\
Params & 7 & 14 & 21 \\
FLOPS & 16 & 30 & 44 \\
\hline \hline AutoDispNet & C & CS & CSS \\
EPE & 2.85 & 2.30 & 2.14 \\
Params & 37 & 74 & 111 \\
FLOPS & 61 & 110 & 160 \\
\hline \hline AutoDispNet-BOHB & C & CS & CSS \\
EPE & $\mathbf{2 . 6 6}$ & $\mathbf{2 . 1 4}$ & $\mathbf{1 . 9 7}$ \\
Params & 37 & 74 & 111 \\
FLOPS & 61 & 110 & 160 \\
\hline
\end{tabular}

Table 2: Performance across the stack. We show improved performance of AutoDispNet architectures across the network stack. End point errors are reported for the Sintel dataset. AutoDispNet-CS matches the baseline performance of three networks with only a single refinement network. The AutoDispNet-BOHB-CS variant outperforms the three network baseline in the second network itself.

optimization also carry over to the large stacked networks. There is a significant improvement with both the architecture optimization and the hyperparameter optimization. The results also reveal that a stack of two networks is already more accurate than a stack of three networks with the baseline. Also the small version of AutoDispNet-css is competitive with DispNet-CS, but runs with 3 times less FLOPS.

Comparison to the state of the art. Although we considered only a limited set of published architectural choices for AutoDispNet, Table 3 reveals that it is competitive with the state of the art on the common public benchmarks. Only PSMNet with its coarse-to-fine strategy performs better on KITTI 2012, but worse on KITTI 2015.

\subsection{Applicability to other tasks}

We also tested our approach on single view depth estimation, another dense prediction task and compare with Laina et al. [42], a state-of-the art single view depth estimation method. The results are shown in Table 4 , On SUN3D we obtain an improvement over the baseline, however the results on NYU dataset are slightly worse. For more details please see the supplement.

\section{Conclusion}

AutoDispNet extends efficient neural architecture search to large-scale dense prediction tasks, in particular U-Netlike architectures. It also leverages hyperparameter tuning by running $\mathrm{BOHB}$ on the selected architecture. Re-

\begin{tabular}{|c|c|c|c|c|c|}
\hline \multirow[t]{2}{*}{ Method } & \multirow{2}{*}{$\begin{array}{c}\text { Sintel } \\
\text { (clean) } \\
\text { AEE } \\
\text { train }\end{array}$} & \multicolumn{2}{|c|}{$\begin{array}{l}\text { KITTI } \\
(2012)\end{array}$} & \multicolumn{2}{|c|}{$\begin{array}{l}\text { KITTI } \\
(2015)\end{array}$} \\
\hline & & $\begin{array}{l}\mathrm{AEE} \\
\text { train }\end{array}$ & $\begin{array}{c}\text { Out-noc } \\
\text { test }\end{array}$ & $\begin{array}{l}\mathrm{AEE} \\
\text { train }\end{array}$ & $\begin{array}{c}\text { D1-all } \\
\text { test }\end{array}$ \\
\hline \multicolumn{6}{|l|}{ Others } \\
\hline SGM [31] & 19.62 & 10.06 & - & 7.21 & $10.86 \%$ \\
\hline DispNet-CSS [37] & 2.33 & 1.40 & - & 1.37 & - \\
\hline DispNet-CSS-ft 37 & 5.53 & $(0.72)$ & $1.82 \%$ & $(0.71)$ & $2.19 \%$ \\
\hline iResNet-i2 [44] & - & - & $1.71 \%$ & - & - \\
\hline EdgeStereo 66 & - & - & - & - & $\mathbf{2 . 1 6} \%$ \\
\hline PSMNet 16 & - & - & $1.49 \%$ & - & $2.32 \%$ \\
\hline GC-Net 39] & - & - & $1.77 \%$ & - & $2.87 \%$ \\
\hline SegStereo 72 & - & - & $1.68 \%$ & - & $2.25 \%$ \\
\hline \multicolumn{6}{|l|}{ Ours } \\
\hline AutoDispNet-css & 2.53 & 1.03 & - & 1.19 & - \\
\hline AutoDispNet-CSS & 2.14 & 0.93 & - & 1.14 & - \\
\hline AutoDispNet-BOHB-CSS & 1.97 & 0.94 & - & 1.15 & - \\
\hline AutoDispNet-BOHB-CSS-ft & 10.55 & $(0.45)$ & $1.70 \%$ & $(0.50)$ & $2.18 \%$ \\
\hline
\end{tabular}

Table 3: Benchmark results. We compare performance of our networks on Sintel and KITTI datasets. For Sintel and KITTI train sets, we report the average end-point error (AEE). Out-noc and D1-all are metrics used to rank methods on the KITTI' 12 and KITTI'15 leader boards. Out-noc is the percentage of outliers exceeding an error threshold of 3px. D1-all is the same metric but applied on all regions (occ and non-occ). Entries enclosed by parentheses indicate if they were finetuned for the evaluated dataset. On KITTI' 15 we are comparable to our baseline. On KITTI' 12 we outperform the baseline with a significant margin.

\begin{tabular}{|c|c|c|c|c|c|}
\hline Method & Params & Abs. rel & Sqr. rel & Rmse & Rmse (log) \\
\hline \multicolumn{6}{|l|}{$S U N 3 D$} \\
\hline Laina et al. [42] & $63 \mathrm{M}$ & 0.272 & 0.248 & 0.703 & 0.500 \\
\hline AutoDepth-S & $63 \mathrm{M}$ & 0.234 & 0.202 & 0.602 & 0.453 \\
\hline AutoDepth-s & $38 \mathrm{M}$ & 0.234 & 0.210 & 0.614 & 0.518 \\
\hline \multicolumn{6}{|l|}{ NYU-Depth-V2 } \\
\hline Laina et al. & $63 \mathrm{M}$ & 0.127 & - & 0.573 & 0.195 \\
\hline AutoDepth-BOHB-S & $63 \mathrm{M}$ & 0.170 & 0.141 & 0.599 & 0.216 \\
\hline
\end{tabular}

Table 4: Results on single view depth estimation. AutoDepth represents a network found using Dense-DARTS. (For details about the metrics see [22])

sults show that this sort of optimization leads to substantial improvements over a manually optimized baseline and reaches state-of-the-art performance on the well-optimized task of disparity estimation. This optimization did not require a huge compute center but was run on common compute hardware, i.e., it can be run by everybody. The total time taken to obtain the AutoDispNet-BOHB-CSS architecture is approximately 42 GPU days.

\section{Acknowledgements}

This study was supported by the German Federal Ministry of Education and Research via the project DeToL and by the German Research Foundation under Germany's Excellence Strategy (CIBSS-EXC-2189). 


\section{References}

[1] Karim Ahmed and Lorenzo Torresani. Maskconnect: Connectivity learning by gradient descent. In $E C C V(5)$, volume 11209 of Lecture Notes in Computer Science, pages 362378. Springer, 2018.

[2] Md Amirul Islam, Mrigank Rochan, Neil D. B. Bruce, and Yang Wang. Gated feedback refinement network for dense image labeling. In Computer Vision and Pattern Recognition (CVPR), 2017.

[3] Vijay Badrinarayanan, Alex Kendall, and Roberto Cipolla. Segnet: A deep convolutional encoder-decoder architecture for image segmentation. IEEE Transactions on Pattern Analysis and Machine Intelligence, 39(12):2481-2495, 2017.

[4] Bowen Baker, Otkrist Gupta, Nikhil Naik, and Ramesh Raskar. Designing neural network architectures using reinforcement learning. In International Conference on Learning Representations, 2017.

[5] Bowen Baker, Otkrist Gupta, Ramesh Raskar, and Nikhil Naik. Accelerating Neural Architecture Search using Performance Prediction. In International Conference on Learning Representations, 2017.

[6] Irwan Bello, Barret Zoph, Vijay Vasudevan, and Quoc V. Le. Neural optimizer search with reinforcement learning. In Doina Precup and Yee Whye Teh, editors, Proceedings of the 34th International Conference on Machine Learning, volume 70 of Proceedings of Machine Learning Research, pages 459-468, International Convention Centre, Sydney, Australia, 06-11 Aug 2017. PMLR.

[7] Gabriel Bender, Pieter-Jan Kindermans, Barret Zoph, Vijay Vasudevan, and Quoc V. Le. Understanding and simplifying one-shot architecture search. In Jennifer Dy and Andreas Krause, editors, Proceedings of the 35th International Conference on Machine Learning, volume 80 of Proceedings of Machine Learning Research, pages 550-559, Stockholmsmässan, Stockholm Sweden, 10-15 Jul 2018. PMLR.

[8] James Bergstra, Daniel Yamins, and David Cox. Making a science of model search: Hyperparameter optimization in hundreds of dimensions for vision architectures. In Sanjoy Dasgupta and David McAllester, editors, Proceedings of the 30th International Conference on Machine Learning, volume 28 of Proceedings of Machine Learning Research, pages 115-123, Atlanta, Georgia, USA, 17-19 Jun 2013. PMLR.

[9] James S. Bergstra, Rémi Bardenet, Yoshua Bengio, and Balázs Kégl. Algorithms for hyper-parameter optimization. In J. Shawe-Taylor, R. S. Zemel, P. L. Bartlett, F. Pereira, and K. Q. Weinberger, editors, Advances in Neural Information Processing Systems 24, pages 2546-2554. Curran Associates, Inc., 2011.

[10] Andrew Brock, Theo Lim, J.M. Ritchie, and Nick Weston. SMASH: One-shot model architecture search through hypernetworks. In International Conference on Learning Representations, 2018.

[11] Daniel J. Butler, Jonas Wulff, Garrett B. Stanley, and Michael J. Black. A naturalistic open source movie for optical flow evaluation. In Computer Vision - ECCV 2012, pages
611-625, Berlin, Heidelberg, 2012. Springer Berlin Heidelberg.

[12] Han Cai, Tianyao Chen, Weinan Zhang, Yong Yu, and Jun Wang. Efficient architecture search by network transformation. In AAAI, pages 2787-2794. AAAI Press, 2018.

[13] Han Cai, Tianyao Chen, Weinan Zhang, Yong Yu, and Jun Wang. Reinforcement learning for architecture search by network transformation. In $A A A I, 2018$.

[14] Han Cai, Jiacheng Yang, Weinan Zhang, Song Han, and Yong Yu. Path-level network transformation for efficient architecture search. CoRR, abs/1806.02639, 2018.

[15] Han Cai, Ligeng Zhu, and Song Han. ProxylessNAS: Direct neural architecture search on target task and hardware. In International Conference on Learning Representations, 2019.

[16] Jia-Ren Chang and Yong-Sheng Chen. Pyramid stereo matching network. arXiv preprint arXiv:1803.08669, 2018.

[17] Liang-Chieh Chen, Maxwell D. Collins, Yukun Zhu, George Papandreou, Barret Zoph, Florian Schroff, Hartwig Adam, and Jonathon Shlens. Searching for efficient multi-scale architectures for dense image prediction. In Annual Conference on Neural Information Processing Systems 2018, NeurIPS 2018, 3-8 December 2018, Montréal, Canada., pages 8713-8724, 2018.

[18] Liang-Chieh Chen, George Papandreou, Florian Schroff, and Hartwig Adam. Rethinking atrous convolution for semantic image segmentation. CoRR, abs/1706.05587, 2017.

[19] Liang-Chieh Chen, Yukun Zhu, George Papandreou, Florian Schroff, and Hartwig Adam. Encoder-decoder with atrous separable convolution for semantic image segmentation. In ECCV (7), volume 11211 of Lecture Notes in Computer Science, pages 833-851. Springer, 2018.

[20] Tobias Domhan, Jost Tobias Springenberg, and Frank Hutter. Speeding up automatic hyperparameter optimization of deep neural networks by extrapolation of learning curves. IJCAI' 15.

[21] Alexey Dosovitskiy, Phillip Fischer, Eddy Ilg, Phillip Häusser, Caner Hazırbaş, Vladamir Golkov, Patrick van der Smagt, Daniel Cremers, and Thomas Brox. Flownet: Learning optical flow with convolutional networks. In IEEE International Conference on Computer Vision (ICCV), 2015.

[22] David Eigen, Christian Puhrsch, and Rob Fergus. Depth map prediction from a single image using a multi-scale deep network. In NIPS, 2014.

[23] Thomas Elsken, Jan Hendrik Metzen, and Frank Hutter. Simple And Efficient Architecture Search for Convolutional Neural Networks. In NIPS Workshop on Meta-Learning, Nov. 2017.

[24] Thomas Elsken, Jan Hendrik Metzen, and Frank Hutter. Neural architecture search. In Hutter et al. [35], pages 69-86. In press, available at http://automl.org/book.

[25] Thomas Elsken, Jan Hendrik Metzen, and Frank Hutter. Efficient multi-objective neural architecture search via lamarckian evolution. In International Conference on Learning Representations, 2019.

[26] Stefan Falkner, Aaron Klein, and Frank Hutter. BOHB: Robust and efficient hyperparameter optimization at scale. In Jennifer Dy and Andreas Krause, editors, Proceedings 
of the 35th International Conference on Machine Learning, volume 80 of Proceedings of Machine Learning Research, pages 1437-1446, Stockholmsmässan, Stockholm Sweden, 10-15 Jul 2018. PMLR.

[27] Matthias Feurer and Frank Hutter. Hyperparameter optimization. In Frank Hutter, Lars Kotthoff, and Joaquin Vanschoren, editors, AutoML: Methods, Sytems, Challenges, chapter 1, pages 3-37. Springer, Dec. 2018.

[28] Mostafa Gamal, Mennatullah Siam, and Moemen AbdelRazek. Shuffleseg: Real-time semantic segmentation network. CoRR, abs/1803.03816, 2018.

[29] Kaiming He, Xiangyu Zhang, Shaoqing Ren, and Jian Sun. Spatial pyramid pooling in deep convolutional networks for visual recognition. In ECCV (3), volume 8691 of Lecture Notes in Computer Science, pages 346-361. Springer, 2014.

[30] Heiko Hirschmuller. Stereo processing by semiglobal matching and mutual information. IEEE Trans. Pattern Anal. Mach. Intell., 30(2), Feb. 2008.

[31] Heiko Hirschmüller. Stereo processing by semiglobal matching and mutual information. PAMI, 30(2):328-341, 2008.

[32] Gao Huang, Zhuang Liu, Laurens van der Maaten, and Kilian Q Weinberger. Densely connected convolutional networks. In Proceedings of the IEEE Conference on Computer Vision and Pattern Recognition, 2017.

[33] Frank Hutter, Holger Hoos, and Kevin Leyton-Brown. An evaluation of sequential model-based optimization for expensive blackbox functions. In Proceedings of GECCO13 Workshop on Blackbox Optimization Benchmarking (BBOB'13), July 2013.

[34] Frank Hutter, Holger Hoos, and Kevin Leyton-Brown. An efficient approach for assessing hyperparameter importance. In Eric P. Xing and Tony Jebara, editors, Proceedings of the 31st International Conference on Machine Learning, volume 32 of Proceedings of Machine Learning Research, pages 754-762, Bejing, China, 22-24 Jun 2014. PMLR.

[35] Frank Hutter, Lars Kotthoff, and Joaquin Vanschoren, editors. Automatic Machine Learning: Methods, Systems, Challenges. Springer, 2018. In press, available at http://automl.org/book.

[36] Eddy Ilg, Nikolaus Mayer, Tonmoy Saikia, Margret Keuper, Alexey Dosovitskiy, and Thomas Brox. Flownet 2.0: Evolution of optical flow estimation with deep networks. In IEEE Conference on Computer Vision and Pattern Recognition (CVPR), 2017.

[37] Eddy Ilg, Tonmoy Saikia, Margret Keuper, and Thomas Brox. Occlusions, motion and depth boundaries with a generic network for disparity, optical flow or scene flow estimation. In European Conference on Computer Vision (ECCV), 2018.

[38] Kevin Jamieson and Ameet Talwalkar. Non-stochastic best arm identification and hyperparameter optimization. In Arthur Gretton and Christian C. Robert, editors, Proceedings of the 19th International Conference on Artificial Intelligence and Statistics, volume 51 of Proceedings of Machine Learning Research, pages 240-248, Cadiz, Spain, 0911 May 2016. PMLR.

[39] Alex Kendall, Hayk Martirosyan, Saumitro Dasgupta, Peter Henry, Ryan Kennedy, Abraham Bachrach, and Adam Bry.
End-to-end learning of geometry and context for deep stereo regression. In Proceedings of the International Conference on Computer Vision (ICCV), 2017.

[40] Diederik P. Kingma and Jimmy Ba. Adam: A method for stochastic optimization. In International Conference on Learning Representations, 2015.

[41] Alex Krizhevsky. Learning multiple layers of features from tiny images. 2009.

[42] Iro Laina, Christian Rupprecht, Vasileios Belagiannis, Federico Tombari, and Nassir Navab. Deeper depth prediction with fully convolutional residual networks. In $3 D$ Vision (3DV), pages 239-248, 2016.

[43] Lisha Li, Kevin Jamieson, Giulia DeSalvo, Afshin Rostamizadeh Rostamizadeh, and Ameet Talwalkar. Hyperband: A novel bandit-based approach to hyperparameter optimization. Journal of Machine Learning Research, 18:1-52, 04 2018.

[44] Zhengfa Liang, Yiliu Feng, Yulan Guo, Hengzhu Liu, Wei Chen, Linbo Qiao, Li Zhou, and Jianfeng Zhang. Learning for disparity estimation through feature constancy. 2018.

[45] Chenxi Liu, Liang-Chieh Chen, Florian Schroff, Hartwig Adam, Wei Hua, Alan Yuille, and Li Fei-Fei. Auto-deeplab: Hierarchical neural architecture search for semantic image segmentation. In CVPR, 2019.

[46] Hanxiao Liu, Karen Simonyan, Oriol Vinyals, Chrisantha Fernando, and Koray Kavukcuoglu. Hierarchical representations for efficient architecture search. In International Conference on Learning Representations, 2018.

[47] Hanxiao Liu, Karen Simonyan, and Yiming Yang. DARTS: Differentiable architecture search. In International Conference on Learning Representations, 2019.

[48] Jonathan Long, Evan Shelhamer, and Trevor Darrell. Fully convolutional networks for semantic segmentation. In CVPR, pages 3431-3440. IEEE Computer Society, 2015.

[49] Ilya Loshchilov and Frank Hutter. Sgdr: Stochastic gradient descent with warm restarts. In International Conference on Learning Representations (ICLR) 2017 Conference Track, Apr. 2017.

[50] Wenjie Luo, Alexander G. Schwing, and Raquel Urtasun. Efficient deep learning for stereo matching. In 2016 IEEE Conference on Computer Vision and Pattern Recognition, CVPR 2016, Las Vegas, NV, USA, June 27-30, 2016, pages 56955703, 2016.

[51] Chris J. Maddison, Andriy Mnih, and Yee Whye Teh. The Concrete Distribution: A Continuous Relaxation of Discrete Random Variables. In International Conference on Learning Representations, 2017.

[52] Nikolaus Mayer, Eddy Ilg, Philip Häusser, Philipp Fischer, Daniel Cremers, Alexey Dosovitskiy, and Thomas Brox. A large dataset to train convolutional networks for disparity, optical flow, and scene flow estimation. In 2016 IEEE Conference on Computer Vision and Pattern Recognition, CVPR 2016, Las Vegas, NV, USA, June 27-30, 2016, pages 40404048, 2016.

[53] Risto Miikkulainen, Jason Liang, Elliot Meyerson, Aditya Rawal, Dan Fink, Olivier Francon, Bala Raju, Hormoz 
Shahrzad, Arshak Navruzyan, Nigel Duffy, and Babak Hodjat. Evolving deep neural networks. In Robert Kozma, Cesare Alippi, Yoonsuck Choe, and Francesco Carlo Morabito, editors, Artificial Intelligence in the Age of Neural Networks and Brain Computing. Amsterdam: Elsevier, 2018.

[54] Jiahao Pang, Wenxiu Sun, Jimmy SJ Ren, Chengxi Yang, and Qiong Yan. Cascade residual learning: A two-stage convolutional neural network for stereo matching. In ICCV Workshop on Geometry Meets Deep Learning, Oct 2017.

[55] Hieu Pham, Melody Guan, Barret Zoph, Quoc V. Le, and Jeff Dean. Efficient neural architecture search via parameters sharing. In Jennifer Dy and Andreas Krause, editors, Proceedings of the 35th International Conference on Machine Learning, volume 80 of Proceedings of Machine Learning Research, pages 4095-4104, Stockholmsmässan, Stockholm Sweden, 10-15 Jul 2018. PMLR.

[56] Anurag Ranjan and Michael Black. Optical flow estimation using a spatial pyramid network. In Proceedings IEEE Conference on Computer Vision and Pattern Recognition (CVPR) 2017, Piscataway, NJ, USA, July 2017. IEEE.

[57] Esteban Real, Alok Aggarwal, Yanping Huang, and Quoc V. Le. Regularized evolution for image classifier architecture search. 2018.

[58] Esteban Real, Sherry Moore, Andrew Selle, Saurabh Saxena, Yutaka Leon Suematsu, Jie Tan, Quoc V. Le, and Alexey Kurakin. Large-scale evolution of image classifiers. In Doina Precup and Yee Whye Teh, editors, Proceedings of the 34th International Conference on Machine Learning, volume 70 of Proceedings of Machine Learning Research, pages 29022911, International Convention Centre, Sydney, Australia, 06-11 Aug 2017. PMLR.

[59] Olaf Ronneberger, Phillip Fischer, and Thomas Brox. U-net: Convolutional networks for biomedical image segmentation. In Medical Image Computing and Computer-Assisted Intervention (MICCAI), volume 9351 of $L N C S$, pages 234-241. Springer, 2015. (available on arXiv:1505.04597 [cs.CV]).

[60] Frederic Runge, Danny Stoll, Stefan Falkner, and Frank Hutter. Learning to design RNA. In International Conference on Learning Representations, 2019.

[61] Shreyas Saxena and Jakob Verbeek. Convolutional neural fabrics. In D. D. Lee, M. Sugiyama, U. V. Luxburg, I. Guyon, and R. Garnett, editors, Advances in Neural Information Processing Systems 29, pages 4053-4061. Curran Associates, Inc., 2016.

[62] Akihito Seki and Marc Pollefeys. Sgm-nets: Semi-global matching with neural networks. In 2017 IEEE Conference on Computer Vision and Pattern Recognition, CVPR 2017, Honolulu, HI, USA, July 21-26, 2017, pages 6640-6649, 2017.

[63] Bobak Shahriari, Kevin Swersky, Ziyu Wang, Ryan P. Adams, and Nando de Freitas. Taking the human out of the loop: A review of bayesian optimization. Proceedings of the IEEE, 104:148-175, 2016.

[64] Jasper Snoek, Hugo Larochelle, and Ryan P. Adams. Practical bayesian optimization of machine learning algorithms. In F. Pereira, C. J. C. Burges, L. Bottou, and K. Q. Weinberger, editors, Advances in Neural Information Processing Systems 25, pages 2951-2959. Curran Associates, Inc., 2012.
[65] Jasper Snoek, Oren Rippel, Kevin Swersky, Ryan Kiros, Nadathur Satish, Narayanan Sundaram, Mostofa Patwary, Mr Prabhat, and Ryan Adams. Scalable bayesian optimization using deep neural networks. In Francis Bach and David Blei, editors, Proceedings of the 32nd International Conference on Machine Learning, volume 37 of Proceedings of Machine Learning Research, pages 2171-2180, Lille, France, 07-09 Jul 2015. PMLR.

[66] Xiao Song, $\mathrm{Xu}$ Zhao, Hanwen $\mathrm{Hu}$, and Liangji Fang. Edgestereo: A context integrated residual pyramid network for stereo matching. Asian Conference on Computer Vision, 2018.

[67] Kenneth O. Stanley and Risto Miikkulainen. Evolving neural networks through augmenting topologies. Evolutionary Computation, 10:99-127, 2002.

[68] Deqing Sun, Xiaodong Yang, Ming-Yu Liu, and Jan Kautz. Pwc-net: Cnns for optical flow using pyramid, warping, and cost volume. In $C V P R$, pages 8934-8943. IEEE Computer Society, 2018.

[69] Mingxing Tan, Bo Chen, Ruoming Pang, Vijay Vasudevan, and Quoc V. Le. Mnasnet: Platform-aware neural architecture search for mobile. CoRR, abs/1807.11626, 2018.

[70] Lingxi Xie and Alan L. Yuille. Genetic cnn. 2017 IEEE International Conference on Computer Vision (ICCV), pages 1388-1397, 2017.

[71] Sirui Xie, Hehui Zheng, Chunxiao Liu, and Liang Lin. SNAS: stochastic neural architecture search. In International Conference on Learning Representations, 2019.

[72] Guorun Yang, Hengshuang Zhao, Jianping Shi, Zhidong Deng, and Jiaya Jia. Segstereo: Exploiting semantic information for disparity estimation. In ECCV, 2018.

[73] Jure Zbontar and Yann LeCun. Stereo matching by training a convolutional neural network to compare image patches. Journal of Machine Learning Research, 17:65:1-65:32, 2016.

[74] Arber Zela, Aaron Klein, Stefan Falkner, and Frank Hutter. Towards automated deep learning: Efficient joint neural architecture and hyperparameter search. In ICML $2018 \mathrm{Au}$ toML Workshop, July 2018.

[75] Chris Zhang, Mengye Ren, and Raquel Urtasun. Graph hypernetworks for neural architecture search. In International Conference on Learning Representations, 2019.

[76] Xiangyu Zhang, Xinyu Zhou, Mengxiao Lin, and Jian Sun. Shufflenet: An extremely efficient convolutional neural network for mobile devices. In 2018 IEEE Conference on Computer Vision and Pattern Recognition, CVPR 2018, Salt Lake City, UT, USA, June 18-22, 2018, pages 6848-6856, 2018.

[77] Zhenli Zhang, Xiangyu Zhang, Chao Peng, Xiangyang Xue, and Jian Sun. Exfuse: Enhancing feature fusion for semantic segmentation. In Computer Vision - ECCV 2018 - 15th European Conference, Munich, Germany, September 8-14, 2018, Proceedings, Part X, pages 273-288, 2018.

[78] Zhao Zhong, Junjie Yan, and Cheng-Lin Liu. Practical network blocks design with q-learning. CoRR, abs/1708.05552, 2017.

[79] Zhao Zhong, Jingchen Yan, Wei Wu, Jing Shao, and ChengLin Liu. Practical block-wise neural network architecture generation. 2018. 
[80] Yi Zhu and Shawn D. Newsam. Densenet for dense flow. In 2017 IEEE International Conference on Image Processing, ICIP 2017, Beijing, China, September 17-20, 2017, pages 790-794, 2017.

[81] Barret Zoph and Quoc V. Le. Neural architecture search with reinforcement learning. In International Conference on Learning Representations, 2017.

[82] Barret Zoph, Vijay Vasudevan, Jonathon Shlens, and Quoc V. Le. Learning transferable architectures for scalable image recognition. In $C V P R$, pages 8697-8710. IEEE Computer Society, 2018. 


\section{Supplementary Material}

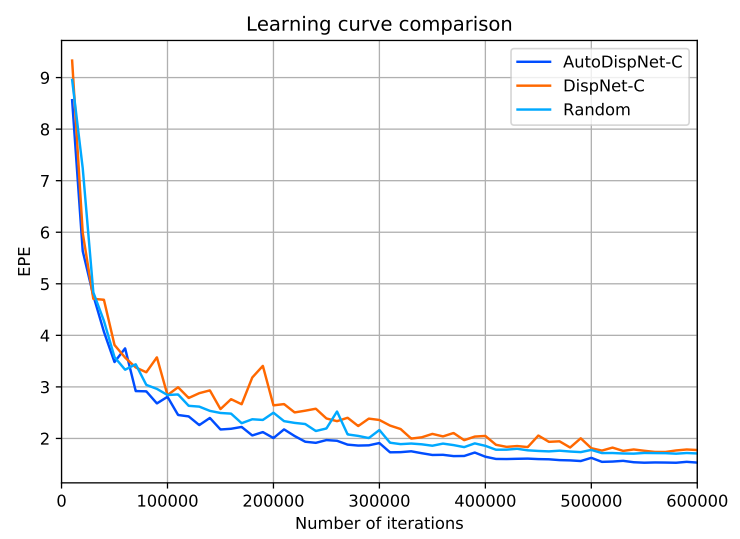

Figure S1: Learning curve comparison. We compare the learning curve of AutoDispNet-C with the baseline DispNet-C and an architecture built with random cells sampled from our search space (denoted by Random). The evolution of EPE over number of iterations is shown for the FlyingThings3D dataset (test split).

\section{Learning curves}

Figure S1 shows the learning curve (evolution of EPE over number of iterations) of AutoDispNet-C. In addition to the baseline (DispNet-C), we also compare the learning curve of a random cell architecture. We randomly sample cells from the search space and stack them in the same fashion as AutoDispNet-C (see section 6 of main paper). We sample four times and build four different random architectures. After training, we pick the best random architecture based on the validation performance on FlyingThings3D. All networks are trained using the same settings as the baseline. Learning curve of the best random architecture is shown in Figure S1. We observe that AutoDispNet-C clearly outperforms the random architecture and the baseline. We also observe that the random architecture is comparable to the baseline. Our observation is similar to Liu et al. [47] on classification, where they also report a surprisingly strong performance for random architectures.

\section{Performance of smaller networks}

We train networks of reduced capacities for both AutoDispNet-C and DispNet-C architectures. For DispNet$\mathrm{C}$ smaller networks are obtained by multiplying the number of channels for each layer by fixed factor (similar to [36]). Smaller variants of AutoDispNet-C are obtained by reduc- ing the number of channels $\left(C_{\text {init }}\right)$ for the first cell. A comparison of EPE vs number of parameters and EPE vs FLOPS is shown in Figure $\mathrm{S} 2$.

\section{Optimizing the refinement network}

\begin{tabular}{|c|c|c|}
\hline \multicolumn{2}{|c|}{ Network stack } & \multirow{2}{*}{$\begin{array}{c}\text { EPE } \\
\text { (Sintel) }\end{array}$} \\
\hline $\mathrm{C}$ & $\mathrm{S}$ & \\
\hline Dense-DARTS & reuse cells & 2.30 \\
\hline Dense-DARTS & Dense-DARTS & 2.32 \\
\hline Dense-DARTS + BOHB & reuse cells + hyperparams & 2.14 \\
\hline Dense-DARTS + BOHB & reuse cells $+\mathrm{BOHB}$ & 2.16 \\
\hline
\end{tabular}

Table S1: We show the results of optimizing cells and hyperparameters of the refinement network in a stack containing two networks (AutoDispNet $\mathrm{C}$ and $\mathrm{S}$ ). First row shows a network where cells for the first network are learned using Dense-DARTS and the refinement network reuses these cells. In the second row, we learn new cell structures of the refinement network using Dense-DARTS. In the third row, we learn cells for the first network and tune the hyperparameters using BOHB. In this case, the refinement network reuses both cells and hyperparameters. In the fourth row, we learn new hyperparameters for the refinement network using BOHB but still use the same cell structures as the first network.

In a stacked setting, the refinement network predicts the residual for correcting errors in predictions from the previous network. Since this task is different from predicting disparity from scratch, we trained a search network to learn specialized cells for the refinement task. However, we found that learning cells for the refinement network did not improve performance over reusing cells learned for the first network. The same argument can also be made for optimizing hyperparameters of the refinement network using BOHB. Surprisingly, even BOHB did not yield improvements over reusing hyperparameters learned for the first network. We show our experimental results in Table $\mathrm{S} 1$. We conjecture that the refinement task is much simpler than estimating disparity from scratch and optimizing cells or hyperparameters is trivial in this case.

\section{Finetuning on the KITTI dataset}

For finetuning on KITTI, we optimize the learning rate and weight decay coefficient using BOHB for the first network in the stack. For running BOHB, we take all samples from KITTI' 12 and KITTI' 15 datasets and use $70 \%$ of the mixture for training. The remaining $30 \%$ of the samples are 


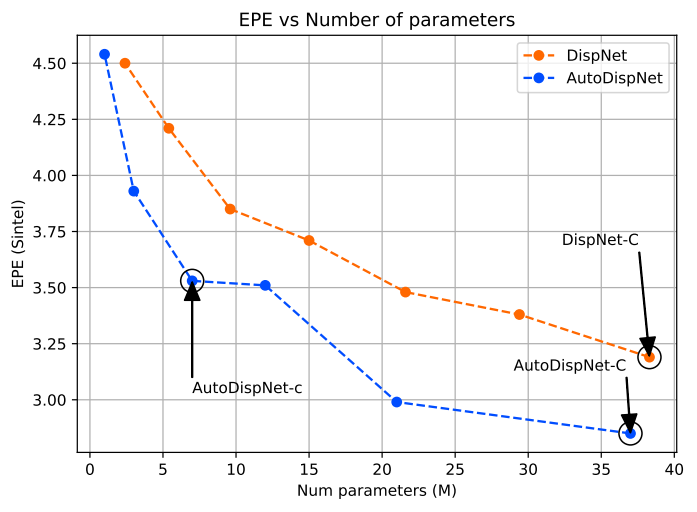

(a) EPE vs Params

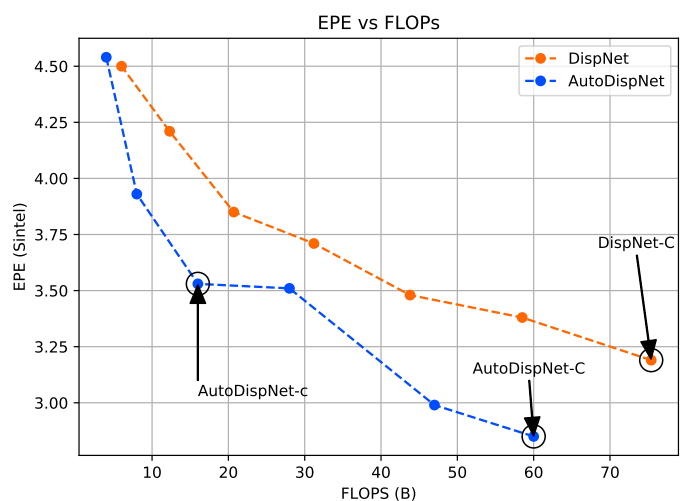

(b) EPE vs FLOPS

Figure S2: Performance of smaller networks. We compare the test performance of smaller DispNet and AutoDispNet architectures. In Figure S2a, we see that AutoDispNet architectures have a lower error with reduced number of parameters compared to the baseline. A similar trend is observed on comparing the EPE with respect to FLOPS ( Figure S2b).

The EPE is shown for the Sintel dataset.

used for validation. We ran BOHB in parallel on 5 GPU workers for a total number of 10 SuccessiveHalving iterations. We used the default BOHB settings with $\eta=3$ and budgets $10 k, 30 k$ and $90 k$ mini-batch iterations. For each budget the learning rate is annealed to zero using a cosine schedule. Figure $\mathrm{S} 3$ shows the EPE of all sampled configurations throughout the optimization procedure. The optimized hyperparameters are then used to finetune the successive networks in the stack. For the last network, we add two more decoding stages to go to full resolution. Here, we use transposed convolutions instead of upsampling cells because applying the cell structure at higher resolutions becomes computationally expensive.

\section{Single view depth estimation}

To evaluate on single view depth estimation, we used the proposed extension of DARTS and compare our results with the competitive method by Laina et al. [42], which uses a ResNet based encoder-decoder with hand-designed upsampling blocks. For a fair comparison, we evaluated both architectures by training them on a subset of the SUN3D dataset using the same hyperparameters and loss function. Please note that in this setting, the siamese part of the network is replaced with a single stream. The extracted architecture is then fine-tuned on $\sim 10,000$ samples from the NYU train dataset using BOHB (optimizing the learning rate and weight decay).

\section{More details on BOHB}

BOHB [26] combines Bayesian Optimization (BO) and Hyperband (HB) [43] in order to exhibit strong anytime and

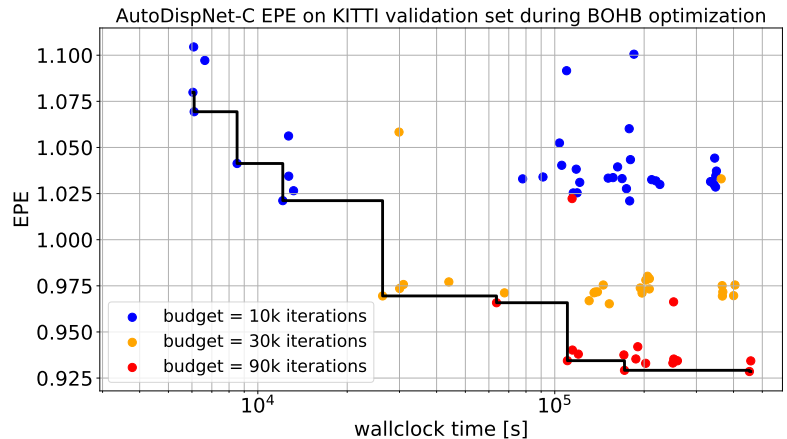

Figure S3: Hyperparameter optimization on KITTI. AutoDispNet-C EPE of all sampled configurations on the different budgets throughout the BOHB optimization procedure. The black line shows the best performing configurations (incumbent) as a function of time.

final performance. BOHB follows the same strategy as HB to allocate resources to configurations calling the SuccessiveHalving ( $\mathrm{SH}$ ) [38] subroutine repeatedly on its inner loop. Refer to Algorithm 1 for a pseudo-code for Hyperband.

On the outerloop HB samples uniformly $N$ random configurations from the hyperparameter search space (lines 3 . 4). Afterwards, $\mathrm{SH}$ evaluates these $N$ configurations (line 7) on the smallest available budget for this outerloop iteration (line 5) and advances the best $1 / \eta$ performing configurations (line 8) to evaluate on a higher budget (increased 


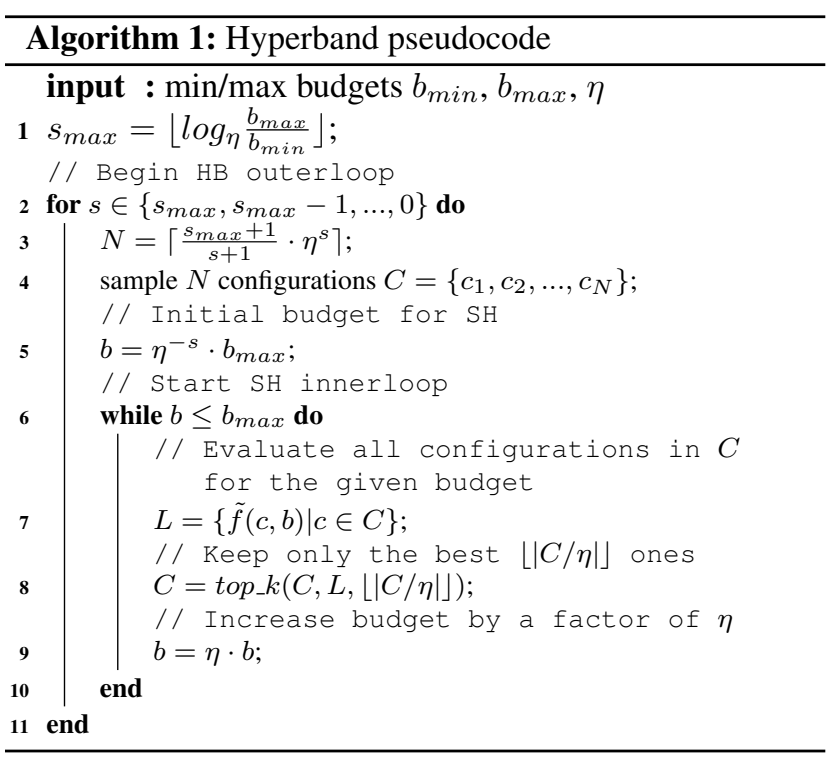

by a factor of $\eta$; line 9). This process goes on until the maximum available budget is reached (line 6. As an example, suppose SH starts with a maximum $N=27$ number of sampled hyperparameter configurations for training a neural network with a minimum budget of $b_{\min }=1$ epoch (first SH innerloop in Figure S4). With an $\eta=3$ the next iteration of $\mathrm{SH}$ would start the best $N / \eta=9$ configurations evaluated on some validation set with the second budget $\eta \cdot b_{\min }=3$ epochs. This will continue until only one configuration is evaluated for $b_{\max }=27$ epochs.

In order to account for the very aggressive evaluations with many configurations on the smallest budget (as done in the first $\mathrm{SH}$ innerloop), $\mathrm{HB}$ resets $\mathrm{SH}$ to start with a smaller degree of aggressiveness, i.e. evaluating the new sampled configurations on a larger initial budget (lines 3 5 in Algorithm 1, illustrated in the second innerloop of Figure S4]. Nevertheless, the number of configurations $N$ sampled in every HB outerloop iteration (line 3 in Algorithm 1 is chosen such that the same total budget is assigned to each $\mathrm{SH}$ run.

Even though BOHB relies on $\mathrm{HB}$ to balance the number of configurations it evaluates and the resources assigned to each configuration, it replaces the random sampling in line 3 of Algorithm 1 by a model-based sampling, where the model is build by the configurations evaluated so far. The strong final performance of BOHB arises from the modelbased guided search, which effectively focuses more attention to regions in space where good configurations lie.

\section{Hyperparameter importance}

In order to assess the importance of hyperparameters over the whole search space we analyze our BOHB results using functional analysis of variance (fANOVA; [34]).

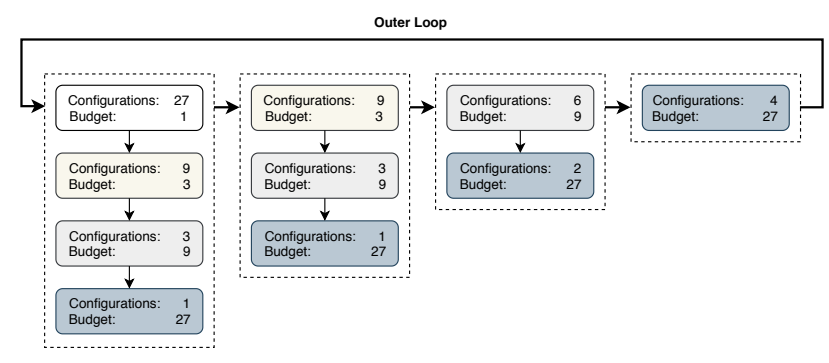

Figure S4: Hyperband inner and outer loops. Hyperband runs SuccessiveHalving on its inner loop with a initial budget and number of starting configurations determined on its outer loop such that the total budget in every SuccessiveHalving run is the same.

This method allows us to quantify how much of the performance variance in the configuration space is explained by single hyperparameters, by marginalizing performances over all possible values that other hyperparameters could have taken. These estimates stem from a random forest model fit on all configurations evaluated on specific budgets during the BOHB optimization procedure.

For the hyperparameter optimization conducted on the FlyingThings3D dataset we observe from Figure S5 that the learning rate remains much more important than the weight decay across the first two budgets ( $16 k$ and $50 k$ iterations). For the highest budget of $150 k$ iterations, the importance of the weight decay hyperparameter becomes larger, however it is still dominated by the learning rate. Notice the optimal value that BOHB determines for each hyperparameter in our space (gray dashed line in Figure [S5). Interestingly, for smaller budgets (i.e. less training iterations) AutoDispNet-C models trained with a small learning rate and high weight decay value (this has a small importance though) perform better on average. As the budget increases the a higher learning rate and a smaller weight decay value are preferred.

We observe similar results when optimizing the learning rate and weight decay for AutoDispNet-C on the KITTI dataset. From the plots in Figure S6 we can see that the learning rate has a higher contribution to the total performance variance throughout all budgets compared to weight decay. However, the optimal values for these two hyperparameters, as determined by $\mathrm{BOHB}$, remain unchanged across these budgets. 

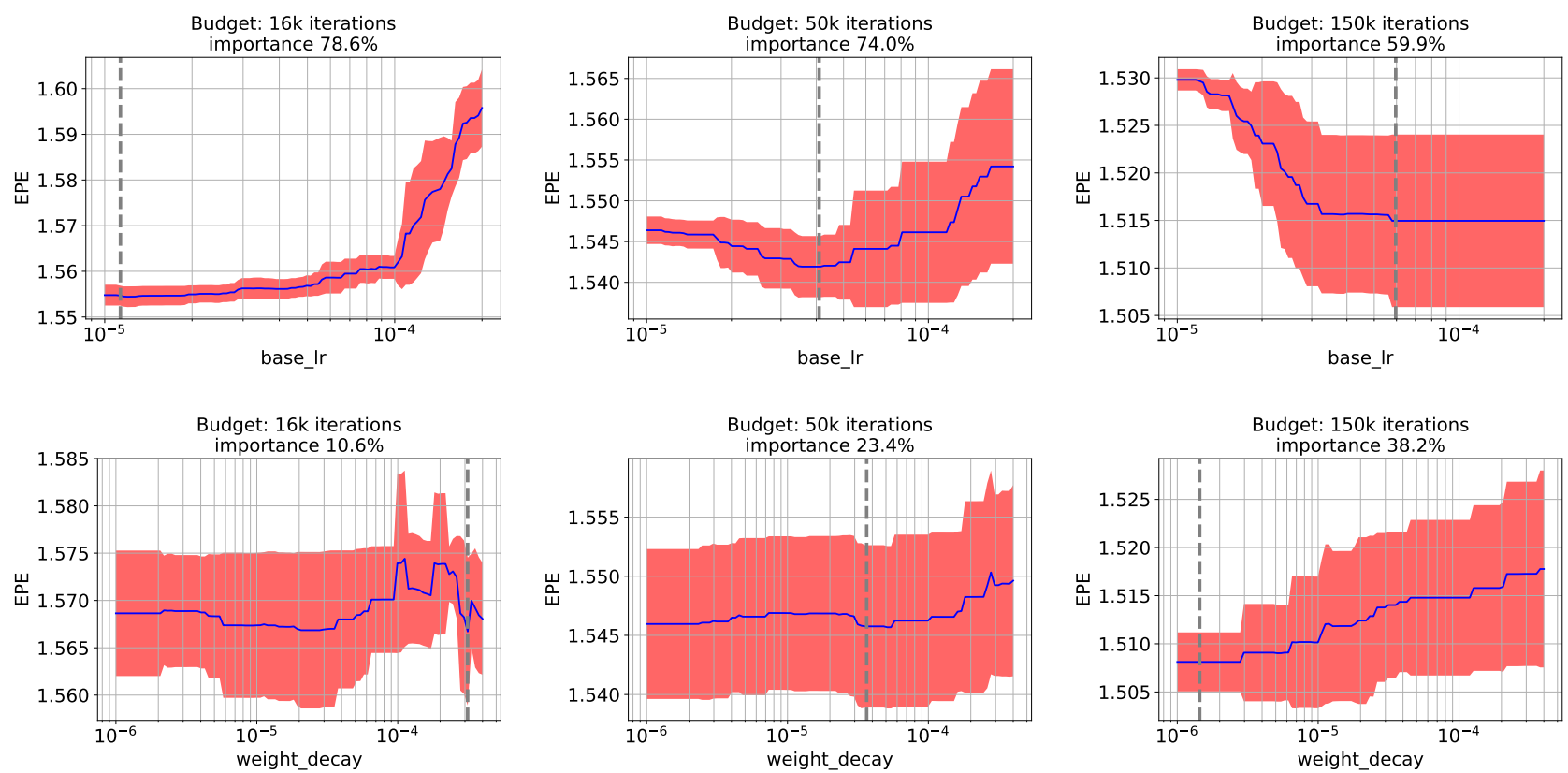

Figure S5: fANOVA plots for all the budgets we run BOHB on the FlyingThings3D dataset. The solid blue line represents the estimated mean EPE (+/- 1std shown by red shaded areas) as a function of hyperparameters as modelled by the random forest we fit to the observations. The importance on top of each plot indicates the fraction of the total variance explained by the individual choice, while the dashed gray line the optimal value as determined by BOHB.
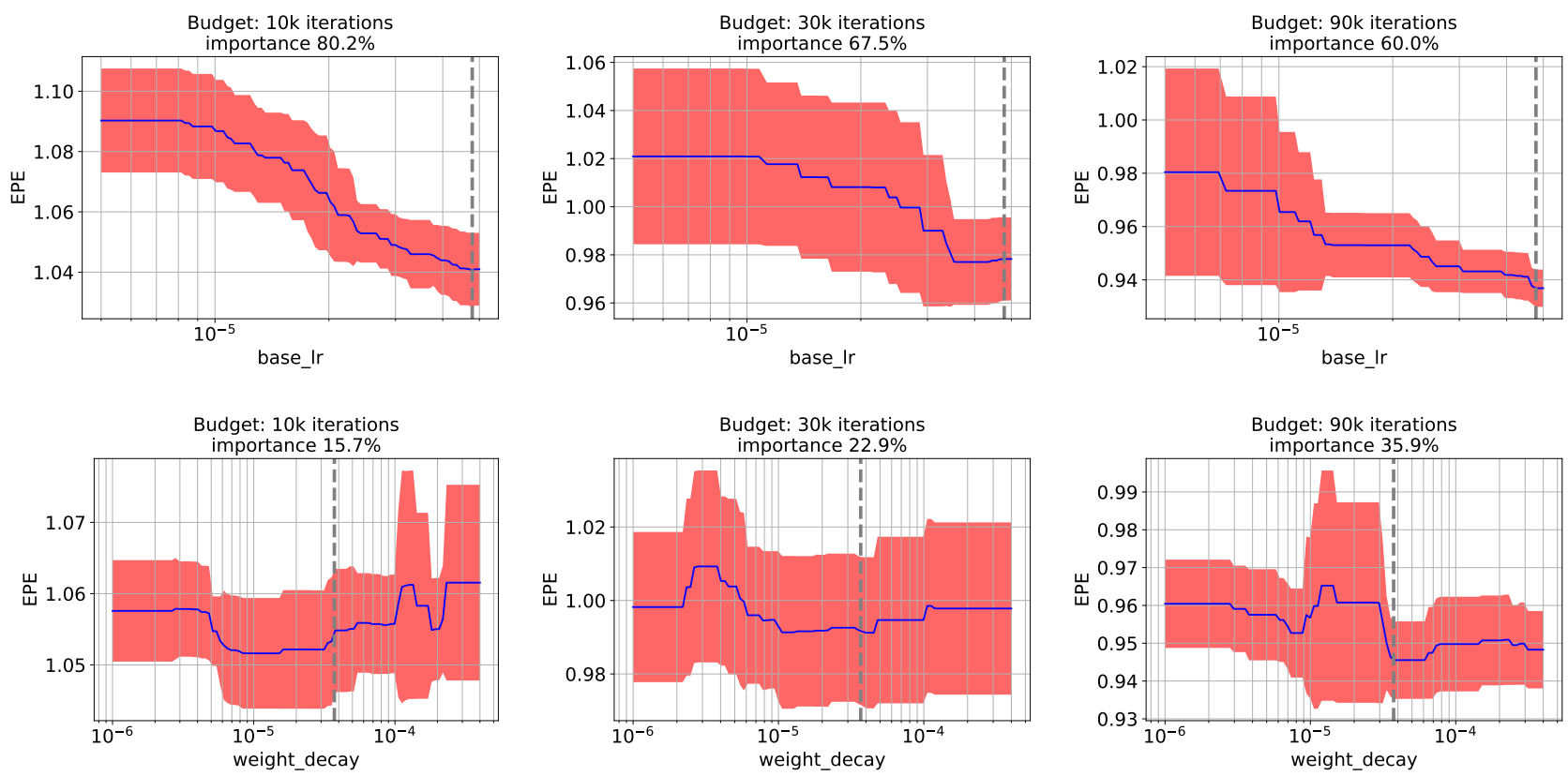

Figure S6: fANOVA plots for all the budgets we run BOHB on the KITTI dataset. The solid blue line represents the estimated mean EPE (+/- 1std shown by red shaded areas) as a function of hyperparameters as modelled by the random forest we fit to the observations. The importance on top of each plot indicates the fraction of the total variance explained by the individual choice, while the dashed gray line the optimal value as determined by BOHB. 